\title{
HILGARDIA
}

JOURNAL OF AGRICULTURAL SCIENCE PUBLISHED BY THE CALIFORNIA AGRICULTURAL EXPERIMENT STATION

Volume 38, Number $14 \cdot$ October, 1967

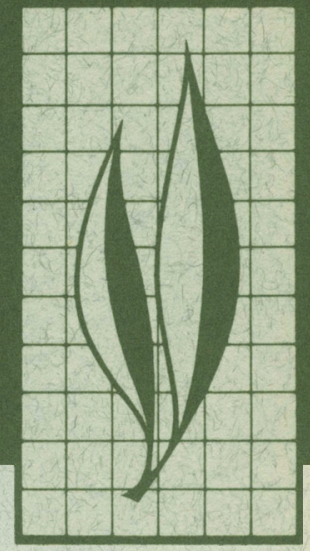

\section{Total Content}

\section{of Nine Mineral Elements in}

\section{Fifty Selected Benchmark Soil Profiles of California}

G. R. Bradford, R. J. Arkley, P. F. Pratt, and F. L. Bair 


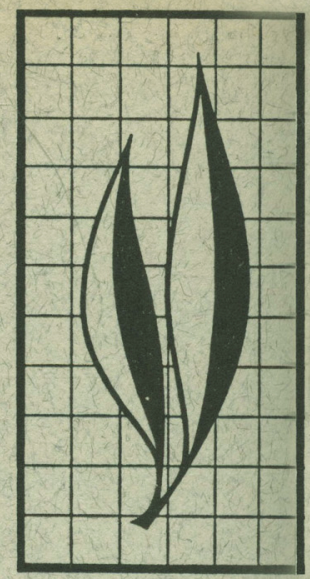

One hundred ninety-five soil horizons from 50 benchmark soil profiles in California were analyzed for total aluminum, magnesium, manganese, nickel, cobalt, copper, iron, molybdenum, and zinc by acid decomposition, resin column separation, and colorimetric methods. Half of the soil series contain low and possibly deficient levels of one or more of the following essential elements: cobalt, copper, molybdenum, manganese, and zinc. Four soil series profiles identified with alkali basins contain relatively high, and probably toxic, levels of molybdenum.

Statistical analyses of the data show that soil series can be grouped on the basis of horizon development, differences between soil profiles or parent material and stratification, or combinations of these controlling influences. In most soils, two distinct groups of elements occur that are negatively correlated between groups and highly correlated positively within groups. Elements which appear most frequently in the same groups together are cobalt, copper, iron, magnesium, and often zinc. Molybdenum is generally negatively correlated with this group of elements.

\section{THE AUTHORS:}

G. R. Bradford is Specialist, Department of Soils and Plant Nutrition, Riverside.

R. J. Arkley is Soil Morphologist in the Experiment Station, and Lecturer, Department of Soils and Plant Nutrition, Berkeley.

P. F. Pratt is Chemist in the Experiment Station, and Professor of Soil Science, Department of Soils and Plant Nutrition, Riverside.

F. L. Bair is Laboratory Technician, Department of Soils and Plant Nutrition, Riverside. 


\section{Total Content of Nine Mineral Elements in Fifty Selected Benchmark Soil Profiles of California ${ }^{1,2}$}

\section{INTRODUCTION}

Swaine's $(1955)^{3}$ Extensive Review of the literature attests to the great variety of analytical techniques used to characterize the trace element status of soilsboth for the total trace element content and for fractions of trace elements, determined by different extractants. The significance of results obtained by diferent techniques - that is, whether by total analysis or by analysis of an extractable fraction-depends upon many variables, such as soil type, parent material, crop, climate, fertilizer, and management practices.

Mitchell (1955, 1963), who has done extensive research on trace elements in soils, concludes that information on total trace element content is exceedingly valuable in indicating excesses or deficiencies. Vink (1963) states that there is a need for continuous research into the productivity of benchmark soils through cooperative efforts of the soil surveyor and soil chemist. The many recent literature references ${ }^{4}$ on the subject of trace elements in soils provide evidence of world-wide interest in this phase of soil science.

The senior author studied a case of severe copper deficiency in orchard grapefruit trees of southern California (Bradford and Harding, 1964), where total copper in the soil was as low as 1.6 ppm. This work stimulated the study reported here of the total content of aluminum, magnesium, nickel, cobalt, copper, iron, molybdenum, manganese, and zine in fifty selected benchmark soil profiles of California.

\section{MATERIALS AND METHODS}

Benchmark soil series samples were selected from an extensive file of soil profile samples in the Department of Soils and Plant Nutrition, Berkeley. The samples were accumulated by cooperative efforts of the University of California, College of Agriculture, and the U. S. Department of Agriculture soil survey teams during more than 50 years of soil survey work in California. A detailed discussion of these soil series is given by Storie and Weir (1953). Each profile sample was selected as the most representative of the series in each area and, in most cases, was undisturbed. They represent some of the major agricultural areas of the State.

\footnotetext{
${ }^{1}$ Submitted for publication March 22, 1967.

${ }^{2}$ This investigation was supported (in part) by Public Health Service Research Grant No. UI 00484 from the National Center for Urban and Industrial Health, and by NSFG 18857.

${ }^{3}$ See "Literature Cited" for citations referred to in the text by author and date.

"See "References."
} 


\section{Brief description of soil series ${ }^{5}$}

The Aiken soils are members of a finetextured kaolinitic, mesic family of Andic Palehumults (Reddish-Brown Lateritic soils) formed on rolling to steep terrain from the weathering of andesitic tuff-breccia under a cover of coniferous forest.

The Cajon (Coachella) soils are members of a sandy-textured, mixed mineralogy, nonacid, thermic family of Typic Xeropsamments (Regosols) formed on hummocky, wind-deposited or wind-modified, lake-laid sands around the northwestern margins of a Pleistocene Salton Sea in the Coachella Valley.

The Fresno soils are members of a fine-textured, mixed mineralogy, thermic family of Typic Nadurargids (Solonetz) formed from moderately coarsetextured, granitic alluvium.

The Hanford soils are members of a coarse, loamy-textured, mixed mineralogy, nonacid, thermic family of Typic Xerorthents (Alluvial soils) formed from recently deposited, moderately coarse-textured, granitic alluvium.

The Holland soils are members of a fine, loamy-textured, mixed mineralogy, mesic family of Typic Palehumults (Reddish-Brown Lateritic soils) formed in weathered, granitic rock on rollingto-steep terrain.

The Hugo soils are members of a fine, loamy-textured, mixed mineralogy, mesic family of Typic Ustochrepts (Regosols) formed on steep, mountainous relief from feldspathic sandstones and shales under dense redwood and Douglas fir forest.

The Imperial soils are members of a fine-textured, mixed, calcareous, thermic family of Typic Torriorthents (Alluvial soils) formed on recent deposits of the lower Colorado River.

The Kettleman soils are members of a fine, loamy-textured, mixed mineralogy, calcareous, thermic family of Typic Xerorthents (Regosols) formed on hillyto-steep relief on calcareous sandstones and shales under a thin cover of annual grass.

The Lassen soils are members of a mesic family of Vertisols (Grumusols) formed on hilly-to-steep relief from basic igneous rocks.

The Los Osos soils are members of a fine-textured, mixed mineralogy, thermic family of Typic Argixerolls (Prairie, or Brunizem soils) found on rollingto-steep slopes under grass vegetation and formed from hard, sedimentary rock.

The Maymen soils are members of a loamy-textured, mixed mineralogy, thermic family of Lithic Ustochrepts (Lithosols) formed under chaparral brush on hard sandstones and shales where the relief is steep and mountainous.

The Merced soils are members of a fine-textured, montmorillonitic, calcareous, thermic Typic Haplaquolls (Humic Gley soils) formed from mainly granitic alluvium but with some mixture of sediment from sedimentary rocks.

The Mojave soils included here are members of a fine, loamy, mixed mineralogy, thermic family of Typic Haplargids (Red Desert soils) mainly formed on granitic alluvium.

The Ramona soils are members of a fine, loamy, mixed mineralogy, thermic family of Typic Haploxeralfs (Noncalcic Brown soils). They are formed on gently sloping alluvial fans of granitic alluvium.

The Redding soils are members of a fine-textured, mixed mineralogy, thermic family of Abruptic Durixeralfs (maximal Noncalcic Brown soils) formed from gravelly alluvium derived from mixed rock sources.

The San Joaquin soils are members of a fine-textured, mixed mineralogy,

${ }^{5}$ The authors express appreciation to Dr. Frederick F. Peterson, Assistant Professor of Soil Science, Department of Soils and Plant Nutrition, University of California, Riverside, for the brief description presented here of soil series amended and annotated to show new U. S. 7th Approximation Soil Classification (1965) names. 
thermic family of Abruptic Durixeralfs (maximal Noncalcic Brown soils) formed on old, gently sloping fans of granitic alluvium.

The Watsonville soils are members of a fine-textured, montmorillonitic, mesic family of Typic Agrialbolls (Planosols) found on coastal terraces about 100 feet above sea level.

The Yolo soils are members of a fine, silty, mixed mineralogy, thermic family of Entic Xerumbrepts (Alluvial soils) formed from recently deposited, medium-textured alluvium derived from sedimentary rocks.

Samples were analyzed by the method of Bradford, et al. (1965). A 0.5-gram soil sample was decomposed with perchloric and hydrofluoric acids. The trace elements were separated and concentrated on a column of a strongly basic anion exchange resin (Dowex 1-X8) by successive elutions, with different volumes of hydrochloric acid of decreasing molarity. Some elements were further separated by organic extraction, and, finally, all elements were determined by conventional colorimetric methods. The entire procedure was considered satisfactory when $100 \pm 5$ per cent of added trace elements were recovered. A wellequipped spectrographic laboratory was also available for these studies. This method of soil analysis was not used, however, because of the complex spectra produced by the usually large amounts of iron, aluminum, manganese, and titanium in soils.

\section{RESULTS AND DISCUSSION}

Table 1 lists the total concentration of aluminum, cobalt, copper, iron, magnesium, molybdenum, manganese, nickel, and zine in each horizon of 50 California soil profiles identified according to soil series classification, depth of horizon, and geographical location.

These data, for the elements known to be essential for plants and/or animals, are significant when compared to the normal concentration ranges compiled by Mitchell (1955) and the deficiency levels as determined by different investigators listed in table 2 .

\begin{tabular}{|c|c|}
\hline $\begin{array}{l}\text { SOIL SERIES } \\
\text { (AND NUMBER) }\end{array}$ & $\begin{array}{c}\text { DEFICIENT } \\
\text { MINERAL EIEMENT }\end{array}$ \\
\hline $\begin{array}{l}\text { Coachella (all) } \\
\text { Fresno ( } 1 \text { of } 3) \\
\text { Mojave }(1 \text { of } 2)\end{array}$ & $\mathrm{Co}$ \\
\hline $\begin{array}{l}\text { Coachella (all) } \\
\text { Fresno ( } 1 \text { of } 3 \text { ) } \\
\text { Holland ( } 2 \text { of } 3 \text { ) } \\
\text { Kettleman }(1 \text { of } 3) \\
\text { Los Osos }(1 \text { of } 3)\end{array}$ & $\mathrm{Cu}$ \\
\hline $\begin{array}{l}\text { Kettleman }(1 \text { of } 3) \\
\text { Maymen }(1 \text { of } 3)\end{array}$ & Mo \\
\hline Kettleman ( 1 of 3 ) & $\mathrm{Mn}$ \\
\hline $\begin{array}{l}\text { Coachella (all) } \\
\text { Fresno ( } 1 \text { of } 3 \text { ) } \\
\text { Hanford ( } 1 \text { of } 2) \\
\text { Kettleman }(1 \text { of } 3) \\
\text { Ramona ( } 1 \text { of } 2)\end{array}$ & $\mathrm{Zn}$ \\
\hline
\end{tabular}

Table 2, then, can be compared with the text table (see left column here) which shows the soil series (from table 1) that are considered low or deficient in the listed mineral elements.

Half of the 50 soil profiles (table 1) might be considered adequately supplied with cobalt, copper, molybdenum, manganese, and zinc. Deficiency symptoms in the other half probably affect plants and/or animals that derive their entire food sources from these soils, and might, therefore, be expected to respond to fertilization with one or more of the essential trace elements.

It is apparent that there is no marked association of total essential trace element content with the series designation. However, since mapping soil series depends entirely on macroscopic observations (aided by a hand lens for an estimate of the mineralogy and rock source of the parent material), it is not surprising that trace-element content is not entirely consistent within widely separated samples of the same soil series. 
TABLE 1

TOTAL CONTENT OF NINE MINERAL ELEMENTS IN 195 HORIZONS OF FIFTY CALIFORNIA SOIL PROFILES

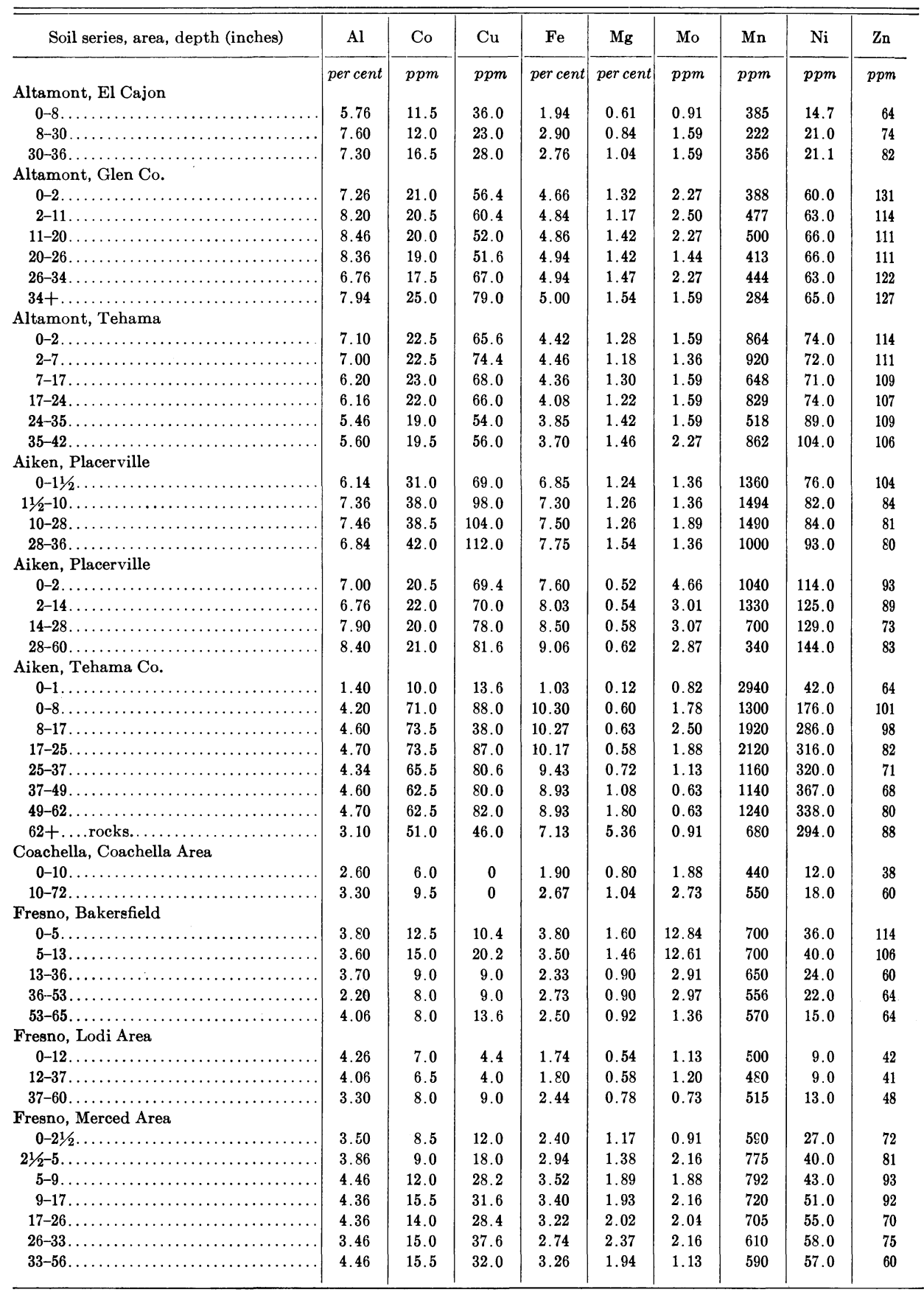


TABle 1-Continued

\begin{tabular}{|c|c|c|c|c|c|c|c|c|c|}
\hline Soil series, area, depth (inches) & $\mathrm{Al}$ & Co & $\mathrm{Cu}$ & $\mathrm{Fe}$ & $\mathrm{Mg}$ & Mo & $\mathrm{Mn}$ & $\mathrm{Ni}$ & $\mathrm{Zn}$ \\
\hline \multicolumn{10}{|l|}{ Hanford, Lodi Area } \\
\hline $0-12 \ldots \ldots \ldots \ldots$ & 4.76 & 8.0 & 12.0 & 2.44 & 0.64 & 1.25 & 540 & 12.0 & 55 \\
\hline $12-38 \ldots \ldots \ldots$ & 4.80 & 9.5 & 11.0 & 2.80 & 0.74 & 3.70 & 515 & 14.0 & 51 \\
\hline $38-72 \ldots \ldots \ldots \ldots \ldots$ & 4.90 & 9.0 & 9.0 & 2.40 & 0.71 & 1.36 & 441 & 12.0 & 51 \\
\hline \multicolumn{10}{|l|}{ Hanford, El Cajon } \\
\hline $0-12 \ldots \ldots \ldots \ldots$ & 4.80 & 14.5 & 11.0 & 4.40 & 1.33 & 1.38 & 950 & 8.0 & 76 \\
\hline $12-72 \ldots \ldots \ldots \ldots \ldots \ldots$ & $4 . \varepsilon 0$ & 16.5 & 13.6 & 4.86 & 1.42 & 1.44 & 1020 & 8.0 & 84 \\
\hline \multicolumn{10}{|l|}{ Holland, Fresno-Sierra } \\
\hline $0-7 \ldots \ldots \ldots \ldots \ldots \ldots \ldots \ldots \ldots$ & 5.26 & 12.8 & 7.0 & 3.44 & 0.66 & 2.98 & 780 & 10.5 & 86 \\
\hline $7-24 \ldots \ldots \ldots \ldots \ldots \ldots \ldots \ldots$ & 5.26 & 13.0 & 6.0 & 3.68 & 0.67 & 3.55 & 615 & 10.5 & 72 \\
\hline $24-60 \ldots$ & 5.16 & 10.0 & 3.6 & 3.48 & 0.58 & 2.95 & 290 & 9.5 & 62 \\
\hline $60+\ldots \ldots \ldots \ldots$ & 5.20 & 10.0 & 1.0 & 3.14 & 0.50 & 3.80 & 245 & 7.3 & 58 \\
\hline \multicolumn{10}{|l|}{ Holland, Placerville } \\
\hline $0-21 / 2 \ldots \ldots \ldots$ & 4.30 & 11.0 & 4.6 & 2.96 & 1.18 & 1.77 & 635 & 21.0 & 74 \\
\hline $21 / 2-12 \ldots$ & 4.90 & 12.0 & 4.6 & 3.20 & 1.42 & 1.88 & 745 & 25.0 & 74 \\
\hline $12-28 \ldots \ldots \ldots \ldots \ldots$ & 4.96 & 14.0 & 9.0 & 3.60 & 1.60 & 1.36 & 631 & 27.0 & 74 \\
\hline $28-48 \ldots \ldots \ldots \ldots \ldots \ldots \ldots \ldots$ & 5.06 & 13.0 & 14.0 & 3.76 & 1.34 & 1.13 & 360 & 25.5 & 60 \\
\hline \multicolumn{10}{|l|}{ Holland, Placerville } \\
\hline $0-3 \ldots \ldots \ldots \ldots$ & 4.56 & 31.5 & 34.4 & 5.40 & 3.70 & 1.55 & 1140 & 168.0 & 89 \\
\hline $3-14 \ldots \ldots \ldots \ldots$ & 5.20 & 40.0 & 52.0 & 6.36 & 4.68 & 1.55 & 1320 & 189.0 & 120 \\
\hline $14-28 \ldots \ldots \ldots \ldots \ldots$ & $4 . \varepsilon 0$ & 36.0 & 49.6 & 5.40 & $3 . \varepsilon 0$ & 1.44 & 1100 & 183.0 & 76 \\
\hline $28-50 \ldots \ldots \ldots \ldots$ & 5.00 & 38.0 & 82.0 & 5.36 & 3.70 & 1.44 & 1000 & 175.0 & 72 \\
\hline \multicolumn{10}{|l|}{ Hugo, Humbolt Co. } \\
\hline $0-11 / 2 \ldots \ldots \ldots \ldots$ & 2.64 & 8.0 & 8.4 & 1. 60 & 0.54 & 1.13 & 2980 & 23.8 & 129 \\
\hline $0-3 \ldots \ldots \ldots \ldots$ & 4.50 & 19.0 & 31.6 & 3.46 & 1.22 & 1.25 & 3320 & 50.5 & 173 \\
\hline $3-13 \ldots$ & 5.00 & 20.5 & 34.4 & 3.58 & 1.40 & 1.55 & 1320 & 46.7 & 123 \\
\hline $13-33 \ldots$ & 4.60 & 18.0 & 39.0 & 3.58 & 1.38 & 1.44 & 950 & 46.3 & 139 \\
\hline $33-52 \ldots \ldots \ldots \ldots$ & 5.00 & 17.5 & 48.8 & 4.26 & 2.02 & 1.55 & 575 & 48.4 & 133 \\
\hline $52-72 \ldots \ldots \ldots \ldots$ & 4.80 & 18.5 & 59.6 & 4.26 & 1.02 & 1.36 & 670 & 48.4 & 130 \\
\hline \multicolumn{10}{|l|}{ Hugo, Dixon Area } \\
\hline $0-10 \ldots \ldots \ldots$ & 5.20 & 19.0 & 64.0 & 4.26 & 1.94 & 1.44 & 620 & 48.4 & 146 \\
\hline $10-26 \ldots \ldots \ldots \ldots$ & 4.80 & 18.5 & 60.0 & 4.56 & 1.05 & 1.36 & 590 & 48.6 & 134 \\
\hline $26-36 \ldots \ldots \ldots \ldots$ & 5.00 & 19.0 & 62.0 & 4.56 & 1.06 & 1.88 & 625 & 53.7 & 151 \\
\hline \multicolumn{10}{|l|}{ Imperial, El Centro } \\
\hline $0-24 \ldots \ldots \ldots \ldots$ & 4.60 & 10.0 & 26.0 & 3.10 & 1.87 & 1.77 & 565 & 27.4 & 100 \\
\hline $21-72 \ldots \ldots \ldots \ldots \ldots$ & 3.40 & 7.0 & 7.0 & 1.80 & 1.21 & 1.13 & 370 & 17.9 & 55 \\
\hline \multicolumn{10}{|l|}{ Imperial, Palo Verde } \\
\hline $0-12 \ldots \ldots \ldots \ldots$ & 4.10 & 9.0 & 24.6 & 2.70 & 1.89 & 1.55 & 535 & 25.4 & 92 \\
\hline $12-56 \ldots \ldots \ldots \ldots$ & 4.50 & 11.0 & 24.0 & 3.10 & 1.94 & 1.36 & 620 & 29.0 & 104 \\
\hline $55-72 \ldots \ldots \ldots \ldots$ & 4.50 & 11.0 & 24.2 & 3.10 & 1.94 & 1.77 & 562 & 27.8 & 98 \\
\hline \multicolumn{10}{|l|}{ Imperial, Brawley } \\
\hline $0-36 \ldots$ & 5.30 & 10.0 & 28.0 & 3.06 & 1.82 & 1.44 & 535 & 28.8 & 101 \\
\hline $36-72$ & 5.54 & 11.0 & 25.6 & 3.10 & 1.81 & 1.44 & 535 & 29.8 & 104 \\
\hline \multicolumn{10}{|l|}{ Kettleman, Mendota } \\
\hline $0-7 \ldots \ldots \ldots \ldots$ & 4.80 & 9.0 & 7.0 & 2.16 & 1.14 & 1.38 & 365 & 71.6 & 63 \\
\hline $7-22$ & 4.30 & 9.0 & 7.0 & 2.03 & 1.24 & 1.18 & 375 & $\varepsilon 0.0$ & 60 \\
\hline $22-30$. & 3.70 & 10.0 & 7.0 & 1.80 & 1.20 & 1.25 & $2 \mathrm{co}$ & 79.0 & 51 \\
\hline $30+\ldots \ldots \ldots \ldots \ldots$ & 4.50 & 9.0 & 7.0 & 2.00 & 1.30 & 1.30 & 320 & 81.2 & 52 \\
\hline \multicolumn{10}{|l|}{ Kettleman, Bakersfiel 1} \\
\hline $0-16 \ldots \ldots \ldots \ldots \ldots$ & 3.26 & 4.0 & 24.0 & 1.76 & 0.79 & 5.80 & 205 & 50.0 & 126 \\
\hline $16-28 \ldots$ & 3.60 & 8.0 & 36.0 & 2.00 & 0.72 & 11.50 & 165 & 65.0 & 178 \\
\hline $28-36 \ldots \ldots \ldots \ldots$ & 4.14 & 6.0 & 52.0 & 2.60 & 0.92 & 13.90 & 170 & 82.0 & 212 \\
\hline Kettleman, Coalinga & & & & & & & & & \\
\hline $0-8 \ldots \ldots \ldots \ldots \ldots$ & 4.60 & 12.5 & 20.4 & 2.60 & 1.19 & 0.83 & 520 & 54.7 & 73 \\
\hline $8-18 \ldots \ldots$ & 4.28 & 12.0 & 15.6 & 2.40 & 0.98 & 0.56 & 500 & 50.0 & 67 \\
\hline $18-40 \ldots \ldots \ldots \ldots$ & 4.45 & 10.0 & 13.6 & 2.50 & 0.96 & 0.78 & 405 & 52.6 & 50 \\
\hline Lassen, Alturas Area & & & & & & & & & \\
\hline $0-5 \ldots \ldots \ldots \ldots \ldots$ & 5.76 & 32.0 & 54.0 & 5.16 & 1.36 & 0.90 & $12 \varepsilon 0$ & 68.8 & 92 \\
\hline $5-12 \ldots \ldots \ldots \ldots$ & 5.40 & 32.0 & 54.0 & 5.24 & 1.50 & 1.13 & 1220 & 73.6 & 90 \\
\hline Lassen, Pixley Area & & & & & & & & & \\
\hline $0-15 \ldots \ldots \ldots \ldots$ & 3.50 & 64.5 & 50.0 & 5.50 & 9.12 & 1.31 & 1300 & 1001 & 88 \\
\hline $15-26 \ldots \ldots \ldots$ & 3.30 & 65.5 & 43.6 & 5.50 & 8.20 & 1.13 & 1190 & 1001 & 81 \\
\hline $26+\ldots \ldots \ldots$ & 1.20 & 78.5 & 14.0 & 4.54 & 16.00 & 0.79 & 355 & 1523 & 61 \\
\hline
\end{tabular}


TABLe 1-Continued

\begin{tabular}{|c|c|c|c|c|c|c|c|c|c|}
\hline Soil series, area, depth (inches) & $\mathrm{Al}$ & Co & $\mathrm{Cu}$ & $\mathrm{Fe}$ & $\mathrm{Mg}$ & Mo & $\mathrm{Mn}$ & $\mathrm{Ni}$ & $\mathrm{Zn}$ \\
\hline Los Osos, Lake Co. & per cent & $p p m$ & $p p m$ & Fer cent & percent & $p p m$ & $p p m$ & $p p m$ & $p p m$ \\
\hline $0-8 \ldots \ldots \ldots \ldots$ & 5.20 & 25.0 & 64.4 & 5.00 & 1.56 & 0.79 & 1380 & 106.6 & 124 \\
\hline $8-20 \ldots$ & 5.40 & 22.0 & 71.0 & 5.68 & 1.38 & 1.13 & 730 & 107.3 & 111 \\
\hline $20-43 \ldots \ldots \ldots \ldots$ & 5.40 & 22.5 & 68.0 & 6.10 & 1.56 & 1.13 & 570 & 108.5 & 107 \\
\hline $43-50 \ldots \ldots \ldots \ldots$ & 5.24 & 17.5 & 62.6 & 5.68 & 1.68 & 1.20 & 550 & 105.2 & 110 \\
\hline $50+\ldots \ldots \ldots \ldots$ & 5.56 & 24.0 & 71.0 & 5.92 & 1.77 & 1.13 & 650 & 113.6 & 129 \\
\hline \multicolumn{10}{|l|}{ Los Osos, Santa Barbara } \\
\hline $0-11 \ldots \ldots \ldots \ldots \ldots$ & 6.00 & 14.5 & 41.0 & 4.42 & 1.12 & 0.56 & 500 & 36.8 & 125 \\
\hline $11-28 \ldots \ldots \ldots \ldots \ldots \ldots \ldots \ldots$ & 5.90 & 12.5 & 40.0 & 4.82 & 1.13 & 0.81 & 504 & 35.7 & 130 \\
\hline $28-41 \ldots \ldots \ldots \ldots \ldots \ldots$ & 6.60 & 15.5 & 43.6 & 5.12 & 1.16 & 1.59 & 500 & 49.4 & 149 \\
\hline $41-53 \ldots \ldots \ldots \ldots \ldots$ & 6.36 & 10.5 & 45.0 & 6.00 & 1.18 & 1.88 & 330 & 32.6 & 160 \\
\hline \multicolumn{10}{|l|}{ Los Osos, Tracy Area } \\
\hline $0-14 \ldots \ldots \ldots \ldots \ldots$ & 3.54 & 6.5 & 0.56 & 1.60 & 0.14 & 2.15 & 500 & 13.6 & 52 \\
\hline $14-31 \ldots \ldots \ldots \ldots \ldots$ & 4.30 & 5.5 & 0.56 & 1.78 & 0.25 & 1.13 & 400 & 18.9 & 56 \\
\hline $31-67 \ldots \ldots \ldots \ldots$ & 4.50 & 8.0 & 0.56 & 2.00 & 0.30 & 1.59 & 135 & 34.2 & 73 \\
\hline \multicolumn{10}{|l|}{ Maymen, Glenn Co. } \\
\hline $0-5 \ldots \ldots \ldots \ldots$ & 7.00 & 17.0 & 58.6 & 6.34 & 1.47 & 1.20 & $7 € 0$ & 75.3 & 172 \\
\hline $5-9 \ldots \ldots \ldots \ldots \ldots$ & 7.90 & 21.0 & 75.6 & 6.00 & 1.78 & 1.59 & 595 & 71.4 & 122 \\
\hline \multicolumn{10}{|l|}{ Maymen, Glenn Co. } \\
\hline $9+$ PM $\ldots \ldots$ & 6.50 & 24.0 & 63.4 & 5.56 & 1.74 & 1.25 & $€ 01$ & 66.6 & 116 \\
\hline \multicolumn{10}{|l|}{ Maymen, Lake Co. } \\
\hline $0-10 \ldots \ldots \ldots \ldots$ & 6.06 & 17.0 & 49.6 & 4.56 & 2.06 & 0.81 & $8 \leq 0$ & 53.6 & 105 \\
\hline $10-20 \ldots \ldots \ldots \ldots \ldots$ & 8.06 & 18.0 & 63.4 & 5.12 & 2.34 & 0.79 & $\varepsilon 00$ & 48.0 & 114 \\
\hline \multicolumn{10}{|l|}{ Maymen, Tehama Co. } \\
\hline $0-1 \ldots \ldots \ldots \ldots \ldots$ & 6.10 & 26.5 & 68.4 & 6.66 & 1.60 & 1.13 & 840 & 98.0 & 116 \\
\hline $1-7 \ldots \ldots \ldots \ldots$ & 6.40 & 28.5 & 83.8 & 6.20 & 1.78 & 1.13 & 601 & 107.0 & 106 \\
\hline $7+\ldots \ldots$ & 6.84 & 26.5 & 89.0 & 6.40 & 2.10 & .68 & 410 & 107.6 & 114 \\
\hline \multicolumn{10}{|l|}{ Merced, Bakersfield } \\
\hline $0-8 \ldots \ldots \ldots \ldots$ & 5.24 & 10.0 & 29.6 & 3.62 & 0.94 & 9.09 & 350 & 21.2 & 78 \\
\hline $8-22 \ldots$ & 6.04 & 11.0 & 30.0 & 4.00 & 1.08 & 4.43 & $2 \varepsilon 0$ & 25.2 & 78 \\
\hline $22-31$. & 5.06 & 8.5 & 18.0 & 2.92 & 0.96 & 4.99 & 215 & 19.0 & 66 \\
\hline $31-52 \ldots$ & 2.94 & 3.5 & 7.0 & 1.30 & 0.36 & 1.13 & 40 & 6.8 & 32 \\
\hline \multicolumn{10}{|l|}{ Merced, Fresno } \\
\hline $0-4 \ldots \ldots \ldots$ & 5.56 & 12.5 & 38.4 & 3.46 & 1.12 & 2.47 & 255 & 67.8 & $\varepsilon 0$ \\
\hline $4-12 \ldots$ & 6.50 & 9.0 & 9.0 & 3.14 & 1.08 & 9.77 & 34 & 66.8 & 93 \\
\hline $12-46$. & 6.30 & 11.0 & 10.0 & 3.20 & 1.18 & 27.50 & 103 & 73.2 & $\mathrm{co}$ \\
\hline $46-56$ & 6.00 & 6.5 & 2.5 & 2.86 & 1.25 & 2.27 & 111 & 61.0 & 92 \\
\hline $56-70 \ldots \ldots \ldots$ & 5.80 & 9.5 & 13.6 & 2.40 & 1.19 & 5.11 & 252 & 44.2 & 73 \\
\hline $70+\ldots \ldots \ldots \ldots \ldots \ldots$ & 4.80 & 8.0 & 7.0 & 1.80 & 1.48 & 11.50 & 412 & 21.0 & 81 \\
\hline \multicolumn{10}{|l|}{ Merced, Merced } \\
\hline $0-5 \ldots \ldots \ldots$ & 6.10 & 4.0 & 0 & 2.46 & $0 . \mathrm{co}_{0}$ & 1.36 & 40 & 20.0 & 120 \\
\hline $5-10 \ldots \ldots \ldots \ldots \ldots$ & 6.20 & 9.0 & 8.2 & 2.70 & 0.84 & 9.09 & 95 & 26.3 & 79 \\
\hline $10-19 \ldots \ldots \ldots \ldots$ & 6.80 & 11.0 & 12.0 & 2.44 & 0.79 & 1.59 & 193 & 25.2 & 65 \\
\hline $19-30 \ldots \ldots \ldots \ldots$ & 6.10 & 8.6 & 10.0 & 2.26 & 0.88 & 2.27 & 211 & 25.4 & 73 \\
\hline $30+\ldots \ldots \ldots \ldots$ & 5.50 & 10.0 & 12.0 & 2.44 & 0.78 & 2.72 & 572 & 50.0 & 62 \\
\hline \multicolumn{10}{|l|}{ Mojave, Barstow } \\
\hline $0-3 \ldots \ldots \ldots \ldots$ & 5.10 & 4.5 & 7.0 & 1.80 & 0.63 & 1.59 & 335 & 10.1 & 55 \\
\hline $3-10 \ldots \ldots \ldots$ & 4.90 & 4.5 & 7.0 & 1.66 & 0.56 & 1.32 & $3 \varepsilon 0$ & 8.2 & 52 \\
\hline $10-20$. & 5.10 & 2.5 & 14.0 & 1.80 & $0 . \varepsilon 0$ & $5 . \varepsilon 0$ & 200 & 8.8 & 52 \\
\hline $20-30$. & 5.00 & 7.2 & 15.0 & 1.94 & 0.81 & 10.07 & $2 \varepsilon 0$ & 10.5 & 54 \\
\hline $30-60 \ldots \ldots \ldots \ldots$ & 5.00 & 2.5 & 5.6 & 1.16 & 0.30 & 0.91 & 193 & 4.2 & 34 \\
\hline \multicolumn{10}{|l|}{ Mojave, Victorville } \\
\hline $0-18 \ldots \ldots \ldots \ldots$ & 5.56 & 9.0 & 18.0 & 2.46 & 0.86 & 5.45 & 445 & 18.9 & 58 \\
\hline $18-30 \ldots \ldots \ldots \ldots$ & 5.46 & 9.5 & 17.6 & 2.44 & 0.94 & 4.77 & 445 & 21.0 & 58 \\
\hline $30-60 \ldots \ldots \ldots \ldots$ & 5.17 & 8.0 & 17.6 & 2.66 & 1.06 & 1.25 & 350 & 21.6 & 73 \\
\hline Ramona, El Cajon & & & & & & & & & \\
\hline $0-10 \ldots \ldots \ldots \ldots$ & 6.70 & 22.5 & 43.6 & 4.16 & 1.58 & 4.43 & 1020 & 12.6 & 73 \\
\hline $10-20 \ldots \ldots \ldots \ldots$ & 6.84 & 22.5 & 49.6 & 5.46 & 1.56 & 2.45 & 1040 & 16.4 & 73 \\
\hline $20-50 \ldots \ldots \ldots \ldots \ldots$ & 6.86 & 18.0 & 38.4 & 4.60 & 1.28 & 2.27 & 860 & 12.2 & 60 \\
\hline $50-72 \ldots \ldots \ldots \ldots \ldots$ & 6.20 & 16.0 & 26.0 & 5.04 & 1.54 & 1.59 & 864 & 11.6 & 64 \\
\hline Ramona, Lodi Area & & & & & & & & & \\
\hline $0-8 \ldots \ldots \ldots \ldots$ & 4.56 & 4.5 & 11.0 & 1.66 & 0.24 & 0.91 & 460 & 14.7 & 34 \\
\hline $8-33 \ldots \ldots \ldots$ & 4.76 & 9.5 & 15.6 & 2.60 & 0.29 & 0.91 & 670 & 24.4 & 44 \\
\hline $33-48 \ldots$. & 5.40 & 2.0 & 13.6 & 2.40 & 0.32 & 5.45 & 365 & 21.0 & 44 \\
\hline $48-72 \ldots \ldots \ldots \ldots \ldots \ldots \ldots \ldots \ldots \ldots \ldots \ldots \ldots \ldots \ldots$ & 6.00 & 9.0 & 14.0 & 2.66 & 0.36 & 8.68 & 640 & 27.0 & 67 \\
\hline
\end{tabular}


TABLE 1-Continued

\begin{tabular}{|c|c|c|c|c|c|c|c|c|c|}
\hline Soil series, area, depth (inches) & $\mathrm{Al}$ & $\mathrm{Co}$ & $\mathrm{Cu}$ & $\mathrm{Fe}$ & $\mathrm{Mg}$ & Mo & $\mathrm{Mn}$ & $\mathrm{Ni}$ & $\mathrm{Zn}$ \\
\hline Redding, Glenn Co. & per cent & ppm & $p p m$ & per cent & percent & ppm & ppm & $p p m$ & $p p m$ \\
\hline $0-7 \ldots \ldots \ldots \ldots$ & 3.30 & 9.0 & 14.0 & 1.86 & 0.32 & 1.14 & 415 & 42.0 & 61 \\
\hline $7-14$ & 3.20 & 9.0 & 16.6 & 1.86 & 0.36 & 5.45 & 430 & 41.0 & 44 \\
\hline $14-23$ & 5.30 & 10.0 & 47.0 & 3.46 & 0.82 & 5.11 & 260 & 91.0 & 90 \\
\hline $23-25 \ldots \ldots \ldots \ldots \ldots$ & 4.90 & 25.0 & 36.0 & 3.66 & 1.22 & 1.32 & 420 & 130.0 & 73 \\
\hline \multicolumn{10}{|l|}{ Redding, Tehama Co. } \\
\hline $0-2 \ldots$ & 3.40 & 9.0 & 17.6 & 1.96 & 0.32 & 4.73 & 350 & 35.0 & 46 \\
\hline $2-7 \ldots$ & 3.20 & 9.0 & 19.0 & 2.00 & 0.32 & 1.59 & 350 & 35.0 & 46 \\
\hline $7-13 \ldots$ & 3.96 & 14.0 & 27.0 & 2.64 & 0.57 & 2.95 & 350 & 49.0 & 43 \\
\hline $13-19$. & 4.44 & 18.0 & 35.4 & 3.64 & 0.79 & 3.70 & 350 & 70.0 & 58 \\
\hline $19-23$ & 4.56 & 19.5 & 40.0 & 4.24 & 1.16 & 1.32 & 370 & 144.0 & 77 \\
\hline $23-35$. . & 4.54 & 38.0 & 40.0 & 3.72 & 1.17 & 4.27 & 576 & 136.0 & 63 \\
\hline $35-48 \ldots \ldots \ldots \ldots \ldots$ & 4.40 & 11.0 & 36.6 & 3.60 & 1.17 & 4.43 & 760 & 72.0 & 62 \\
\hline $48-60 \ldots \ldots \ldots \ldots \ldots$ & 4.54 & 9.5 & 40.0 & 3.90 & 1.22 & 1.14 & 380 & 70.0 & 66 \\
\hline \multicolumn{10}{|l|}{ San Joaquin, Merced } \\
\hline $0-9 \ldots \ldots \ldots \ldots$ & 4.40 & 10.0 & 33.8 & 2.00 & 0.57 & 2.45 & 270 & 17.0 & 52 \\
\hline $9-13 \ldots \ldots \ldots \ldots \ldots \ldots$ & 4.40 & 11.0 & 11.0 & 1.96 & 0.51 & 1.59 & 320 & 18.0 & 43 \\
\hline $13-171 / 2 \ldots \ldots \ldots \ldots \ldots$ & 4.42 & 11.0 & 11.0 & 1.96 & 0.50 & 2.27 & 580 & 20.0 & 44 \\
\hline $171 / 2-201 / 2 \ldots$ & 4.78 & 11.5 & 9.0 & 2.06 & 0.49 & 1.36 & 550 & 20.0 & 44 \\
\hline $201 / 2-25 \ldots \ldots \ldots \ldots \ldots$ & 7.30 & 9.5 & 11.0 & 4.20 & 0.68 & 1.59 & 330 & 37.0 & 64 \\
\hline $25-27 \ldots \ldots \ldots \ldots \ldots \ldots \ldots$ & 5.40 & 11.0 & 14.0 & 3.20 & 1.05 & 1.14 & 220 & 24.0 & 64 \\
\hline $27-40 \ldots \ldots \ldots \ldots \ldots \ldots \ldots$ & 5.04 & 10.5 & 18.0 & 3.80 & 1.40 & 2.93 & 482 & 29.4 & 82 \\
\hline $40-50 \ldots \ldots \ldots \ldots \ldots \ldots$ & 4.66 & 15.5 & 16.0 & 3.24 & 1.06 & 2.93 & 775 & 24.8 & 74 \\
\hline \multicolumn{10}{|l|}{ San Joaquin, Pixley } \\
\hline $0-16 \ldots \ldots \ldots \ldots \ldots$ & 4.14 & 10.5 & 8.6 & 2.76 & 0.68 & 1.77 & 690 & 19.0 & 84 \\
\hline $16-24 \ldots \ldots \ldots \ldots \ldots \ldots$ & 4.70 & 11.0 & 14.0 & 2.70 & 0.80 & 3.22 & 745 & 22.2 & 66 \\
\hline $24-34$ & 5.04 & 12.0 & 14.6 & 3.00 & 1.02 & 2.93 & 630 & 26.0 & 89 \\
\hline $34-66 \ldots \ldots \ldots \ldots \ldots$ & 4.76 & 12.0 & 16.0 & 3.72 & 1.20 & 1.36 & 730 & 23.8 & 81 \\
\hline \multicolumn{10}{|l|}{ Staten, orig. loam } \\
\hline $0-12 \ldots \ldots \ldots \ldots$ & 1.30 & 6.0 & 7.6 & 2.16 & 0.72 & 3.55 & 660 & 22.8 & 28 \\
\hline $12-40 \ldots \ldots \ldots \ldots \ldots$ & 1.36 & 3.5 & 11.0 & 1.04 & 0.54 & 1.32 & 370 & 19.0 & 28 \\
\hline \multicolumn{10}{|l|}{ Watsonville, Santa Barbara } \\
\hline $0-8 \ldots \ldots \ldots \ldots \ldots \ldots \ldots$ & 3.20 & 10.5 & 7.6 & 1.20 & 0.28 & 0.79 & 630 & 27.0 & 32 \\
\hline $8-18 \ldots \ldots \ldots \ldots \ldots$ & 3.06 & 10.5 & 4.4 & 1.24 & 0.24 & 0.44 & 710 & 30.6 & 33 \\
\hline $18-24 \ldots \ldots \ldots \ldots \ldots \ldots$ & 3.30 & 6.0 & 1.6 & 1.00 & 0.16 & 1.36 & 370 & 16.9 & 31 \\
\hline $24-37 \ldots \ldots \ldots \ldots \ldots \ldots$ & 4.06 & 5.0 & 1.6 & 2.90 & 0.60 & 2.50 & 140 & 49.4 & 61 \\
\hline $37-50 \ldots \ldots \ldots \ldots \ldots \ldots$ & 4.70 & 2.5 & 12.4 & 2.84 & 0.70 & 1.89 & 90 & 39.6 & 66 \\
\hline $50-70 \ldots \ldots \ldots \ldots \ldots$ & 4.40 & 4.5 & 13.0 & 2.60 & 0.62 & 1.77 & 100 & 36.4 & 73 \\
\hline \multicolumn{10}{|l|}{ Watsonville, Santa Cruz } \\
\hline $0-10 \ldots \ldots \ldots \ldots \ldots$ & 3.76 & 12.0 & 12.0 & 1.60 & 0.22 & 1.14 & 700 & 21.0 & 49 \\
\hline $10-20 \ldots \ldots \ldots \ldots \ldots \ldots$ & 3.90 & 13.0 & 11.0 & 1.64 & 0.22 & 1.59 & 840 & 23.8 & 48 \\
\hline $20-24 \ldots \ldots \ldots \ldots \ldots$ & 3.76 & 34.5 & 12.0 & 2.50 & 0.26 & 1.78 & 1490 & 25.2 & 52 \\
\hline $24-37 \ldots \ldots \ldots \ldots \ldots \ldots$ & 4.74 & 10.0 & 18.0 & 2.60 & 0.46 & 1.14 & 160 & 33.6 & 72 \\
\hline $37-50 \ldots \ldots \ldots \ldots \ldots$ & 4.84 & 4.5 & 13.0 & 2.44 & 0.46 & 1.44 & 240 & 34.7 & 70 \\
\hline \multicolumn{10}{|l|}{ Watsonville, Santa Cruz } \\
\hline $0-19 \ldots \ldots \ldots \ldots \ldots \ldots$ & 4.14 & 8.5 & 2.0 & 2.16 & 0.30 & 1.36 & 470 & 23.2 & 33 \\
\hline $19-22 \ldots \ldots \ldots \ldots \ldots \ldots$ & 4.44 & 7.0 & 2.0 & 3.04 & 0.41 & 0.68 & 292 & 41.0 & 37 \\
\hline $22-28 \ldots \ldots \ldots \ldots \ldots \ldots \ldots \ldots \ldots \ldots$ & 4.06 & 7.0 & 4.4 & 1.68 & 0.26 & 0.22 & 485 & 16.8 & 31 \\
\hline $28-40 \ldots \ldots \ldots \ldots \ldots \ldots \ldots \ldots \ldots$ & 4.76 & 11.0 & 7.6 & 2.12 & 0.30 & 0.91 & 180 & 35.8 & 32 \\
\hline \multicolumn{10}{|l|}{ Yolo, Dixon Area } \\
\hline $0-15 \ldots \ldots \ldots$ & 5.50 & 22.5 & 36.0 & 3.76 & 2.36 & 0.82 & 712 & 227.0 & 96 \\
\hline $15-72 \ldots \ldots \ldots$ & 5.10 & 16.0 & 36.6 & 3.60 & 2.51 & 2.39 & 325 & 227.0 & 40 \\
\hline \multicolumn{10}{|l|}{ Yolo, Dixon } \\
\hline $0-15 \ldots \ldots$ & 5.20 & 23.0 & 39.0 & 3.60 & 2.56 & 2.50 & 482 & 256.0 & 48 \\
\hline $15-72 \ldots \ldots \ldots$ & 5.20 & 21.0 & 38.4 & 3.66 & 2.49 & 2.27 & 410 & 232.0 & 94 \\
\hline \multicolumn{10}{|l|}{ Yolo, Tehama Co. } \\
\hline $0-11 \ldots \ldots \ldots \ldots$ & 6.06 & 25.5 & 54.0 & 4.24 & 2.44 & 2.04 & 675 & 183.0 & 76 \\
\hline $11-34 \ldots \ldots \ldots \ldots \ldots \ldots \ldots$ & 6.26 & 27.0 & 55.0 & 4.44 & 2.63 & 1.87 & 760 & 200.0 & 69 \\
\hline $34-68 \ldots \ldots \ldots \ldots \ldots \ldots \ldots \ldots \ldots \ldots \ldots \ldots$ & 6.00 & 23.0 & 50.0 & 4.20 & 3.00 & 1.59 & 510 & 200.0 & 59 \\
\hline
\end{tabular}


TABLE 2

NORMAL TOTAL AND DEFICIENT CONCENTRATIONS OF CERTAIN ESSENTIAL TRACE ELEMENTS IN SOIL AS REPORTED IN THE LITERATURE

\begin{tabular}{|c|c|c|c|c|}
\hline \multirow{2}{*}{ Element } & \multicolumn{2}{|c|}{ Normal concentration } & \multicolumn{2}{|r|}{ Deficient concertration } \\
\hline & Total & Reference & Total & Reference \\
\hline & ppm & & $p p m$ & \\
\hline Co... & $1.0-40$ & Mitchell (1955) & $<4-5$ & $\begin{array}{l}\text { Harvey (1937), Patterson (1937), Rigg (1940), Russell (1938), } \\
\text { Stewart, et al. (1941), Walsh, et al. (1956), Kubota (1964) }\end{array}$ \\
\hline $\mathrm{Cu}$. & $2.0-100$ & Mitehell (1955) & $<12$ & $\begin{array}{l}\text { Bould, et al. (195'), Bradford and Harding (1964), Knott (1933), } \\
\text { Purvis and Ragg (1962) }\end{array}$ \\
\hline Mo....... & $0.2-5$ & Mitchell (1955) & $<1$ & Barshad (1951), Robinson, et al. (1951), Walsh, et al. (19:2) \\
\hline Mn....... & $200.0-3,000$ & Mitchell (1955) & $<200$ & Mitchell (1955), Swaine (1955) \\
\hline $\mathrm{Zn}$. & $10.0-300$ & Mitchell (1955) & $<80$ & Thorne, et al. (1942) (see footnote ${ }^{*}$ ) \\
\hline
\end{tabular}

* Eighty per cent of 42 soils analyzed for total and extractable $\mathrm{Zn}$ showed plant response to added $\mathrm{Zn}$ where total Zn was less than $80 \mathrm{ppm}$. From Ph.D. thesis of John Trierweiler, Department of Agronomy, Colorado State University, Fort Collins, Colorado. Thesis in process of publication.

TABLE 3

GROUPING OF SOIL PROFILES ACCORDING TO PARENT MATERIAL AND TRACE ELEMENT CONTENT IN UPPER 20 INCHES OF SOIL

\begin{tabular}{|c|c|c|c|c|c|}
\hline \multirow{3}{*}{ Parent material and soil profile } & \multicolumn{5}{|c|}{ Number of soil profiles containing: } \\
\hline & \multicolumn{2}{|c|}{$\mathrm{Zn}$} & \multicolumn{2}{|c|}{$\mathrm{Cu}$} & \multirow{2}{*}{$\begin{array}{l}\text { Deficient trace } \\
\text { elements }\end{array}$} \\
\hline & $>80 \mathrm{ppm}$ & $<\&_{0} \mathrm{ppm}$ & $>12 \mathrm{ppm}$ & $<12 \mathrm{ppm}$ & \\
\hline \multicolumn{6}{|l|}{ Granitic alluvium: } \\
\hline Coachella........ & & 1 & & 1 & \\
\hline Fresno (alkali) .............. & 2 & 1 & 2 & 1 & \\
\hline 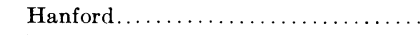 & & 2 & & 2 & \\
\hline Ramona....................... & & 2 & 2 & & \\
\hline San Joaquin ........ & $1^{*}$ & 1 & 2 & & \\
\hline Mojave.................. & & 2 & 1 & 1 & 1-Co \\
\hline \multicolumn{6}{|l|}{ Granitic rock: } \\
\hline Holland ..... & 2 & 1 & 1 & 2 & \\
\hline \multicolumn{6}{|l|}{ Mixed alluvium: } \\
\hline Imperial (recent) & 3 & & 3 & & \\
\hline Merced (young) .... & 2 & 1 & 2 & 1 & $2-\mathrm{Mn}$ \\
\hline Redding (very old).. & & 2 & 2 & & \\
\hline Watsonville (old). & & 3 & & 3 & 2-Mo \\
\hline Yolo (recent) . . . . . . . . & 1 & 2 & 3 & & \\
\hline \multicolumn{6}{|l|}{ Basic igneous rock: } \\
\hline Aiken........... & 3 & & 3 & & \\
\hline Lassen........... & 2 & & 2 & & \\
\hline \multicolumn{6}{|l|}{ Sedimentary rock: } \\
\hline Altamont........ & 2 & 1 & 3 & & \\
\hline Hugo............ & 2 & & 2 & & \\
\hline Los Osos.................. & 2 & & 2 & & 1-Mo \\
\hline Kettleman................. & 1 & 2 & 2 & 1 & 1-Co, 1-Mo \\
\hline 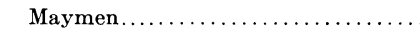 & 3 & & 3 & & 1-Mo \\
\hline Peat soil. & & 1 & & 1 & 1-Co \\
\hline
\end{tabular}

* May not be pure granitic alluvium. 
Table 3 shows the grouping of soil profiles according to parent material, their zinc and copper content, and other low trace-element values. Soils formed from granitic alluvium and granite rock usually can be expected to be low in zine, copper, or both. Two old soils on mixed alluvium are low in zine; one is low in copper. Soils formed from sedimentary rocks are generally well supplied with trace elements, but may vary considerably depending on the sediment. The two basic igneous rock soils show no low values as would be expected from this kind of rock. The peat soil is deficient in zine, copper, and cobalt. In general, the distribution of trace-element content is reasonably consistent within groupings based on soil parent material. This agrees with the work of Archer (1963). Where anomalies exist, the availability of chemical analysis of ber.chmark soil profiles may lead to the identification of a previously unrecognized soil type as discussed by Taylor, et al. (1956).

The Fresno and Kettleman soils from near Bakersfield and the Merced soil from Fresno are all alkali basin soils and contain relatively high, and probably toxic, concentrations of molybdenum. The Mojave soil, an unleached desert soil from Barstow, is also high in molybdenum.

Table 4 shows several highly significant relationships between the total contents in soils of several pairs of elements. Considering the diverse nature of the soil profiles and parent material, some of these relations are notable. Both copper and cobalt tend to increase linearly with increasing iron content. Magnesium content tends to vary inversely with molybdenum, although most soils are relatively low in both molybdenum and magnesium. The highest nickel and magnesium levels are associated with a serpentine soil. In most other soils, magnesium is low, and nickel varies over a wide range.

Table 5 shows the within-group and between-group correlations of groups of elements for several soil series. The data for each series in table 5 were prepared by first arranging the larger values of $r$ into a matrix table (omitting decimals), and then, from the patterns evolved, the elements were separated into groups (labeled A, B, and C) and the withingroup mean correlation coefficient $(\overline{\mathrm{r}})$ and the between-group values of $\overline{\mathrm{r}}$ were calculated. It is evident from table 5 that, in most soils, there are two distinct groups of elements which are negatively correlated between groups and highly correlated positively within groups of elements. In some cases, a third group of elements (C) has correlation coefficients that are intermediate between groups A and $\mathrm{B}$.

The original data in table 1 suggest that the source of these coefficients for the various soil series are due mainly to: horizon development in the Redding, San Joaquin, and Watsonville series; the parent material of different soil profiles in the Altamont, Aiken, Holland, Kettleman and Ramona series; parent material and stratification in the Yolo, Merced, and Imperial series; and parent material and profile development in the Fresno, Hugo, Los Osos, and Mojave series.

The elements which appear most frequently in the same groups in the matrix tables are cobalt, copper, iron, and magnesium, and usually zinc. Essentially the same elements have high correlation coefficients based on analyses of all profiles as listed in table 4. This appears to be primarily a parent-material effect. Molybdenum is generally negatively correlated with this group of elements. 
TABLE 4

ELEMENT CORRELATIONS BASED ON ANALYSIS OF 195 HORIZONS OF 50 BENCHMARK SOIL PROFILES FROM CALIFORNIA

\begin{tabular}{|c|c|c|c|c|c|c|c|c|c|}
\hline & $\mathrm{Al}$ & Co & $\mathrm{Cu}$ & $\mathrm{Fe}$ & $\mathrm{Mg}$ & Mo & $\mathrm{Mn}$ & $\mathrm{Ni}$ & $\mathrm{Zn}$ \\
\hline 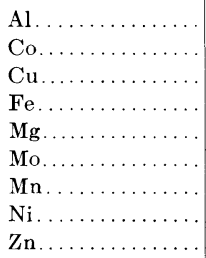 & 1.00 & $\begin{array}{l}0.077 \mathrm{NS} \\
1.000\end{array}$ & $\begin{array}{l}0.52^{* * *} \\
0.63^{* * *} \\
1.00\end{array}$ & $\begin{array}{l}0.45^{* * *} \\
0.78^{* * *} \\
0.82^{* * *} \\
1.00\end{array}$ & $\begin{array}{c}-0.11 \mathrm{NS} \\
0.56^{* * *} \\
0.20^{*} \\
0.25^{* *} \\
1.00\end{array}$ & $\begin{array}{r}0.029 \mathrm{NS} \\
-0.170 \mathrm{NS} \\
-0.140 \mathrm{NS} \\
-0.120 \mathrm{NS} \\
-0.920^{* * *} \\
1.000\end{array}$ & $\begin{array}{c}-0.03 \mathrm{NS} \\
0.49^{* * *} \\
0.34^{* * *} \\
0.41^{* * *} \\
0.11 \mathrm{NS} \\
-0.20^{*} \\
1.00\end{array}$ & $\begin{array}{c}-0.78^{* * *} \\
0.68^{* * *} \\
0.25^{* *} \\
0.33^{* * *} \\
0.84^{* * *} \\
-0.29^{* *} \\
0.15 \mathrm{NS} \\
1.00\end{array}$ & $\begin{array}{l}0.38^{* * *} \\
0.20^{* *} \\
0.53^{* * *} \\
0.42^{* * *} \\
0.15 \mathrm{NS} \\
0.13 \mathrm{NS} \\
0.24^{*} \\
0.10 \mathrm{NS} \\
1.00\end{array}$ \\
\hline
\end{tabular}

* Significant at the 5 per cent (0.19) level.

** Significant at the 1 per cent $(0.25)$ level.

Significant at the 0.1 per cent $(0.32)$ level

$\mathrm{NS}=$ Not significant.

TABLE 5

WITHIN- AND BETWEEN-GROUP CORRELATION OF GROUPS OF ELEMENTS FOR SEVERAL SOIL SERIES

Yolo Series

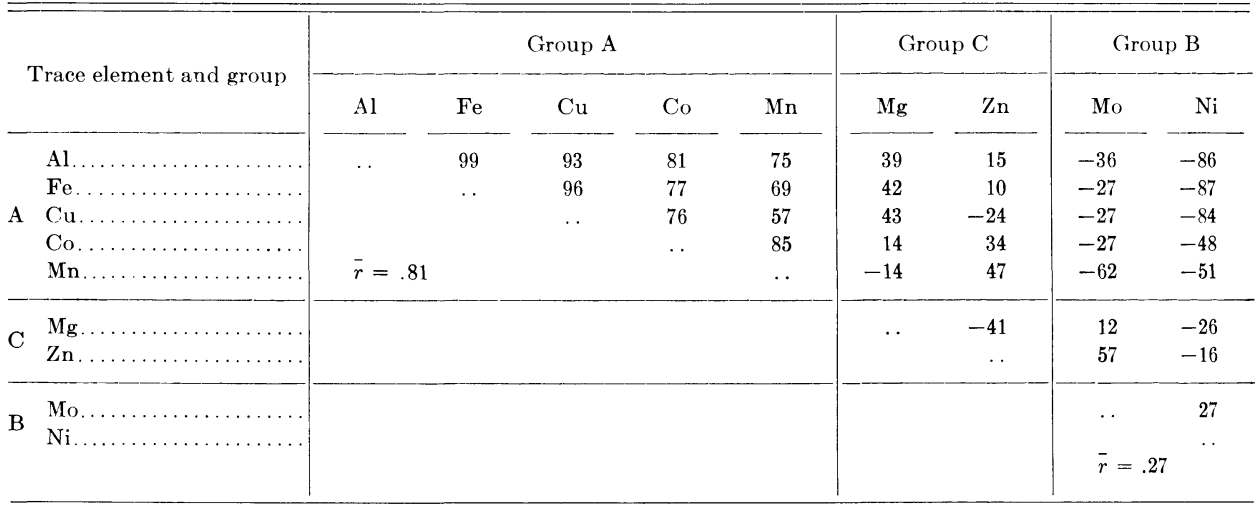

Between $\mathrm{A}-\mathrm{B}, \bar{r}=-.53$; between $\mathrm{Ni}-\mathrm{A}, \bar{r}=.71$.

Watsonville Series

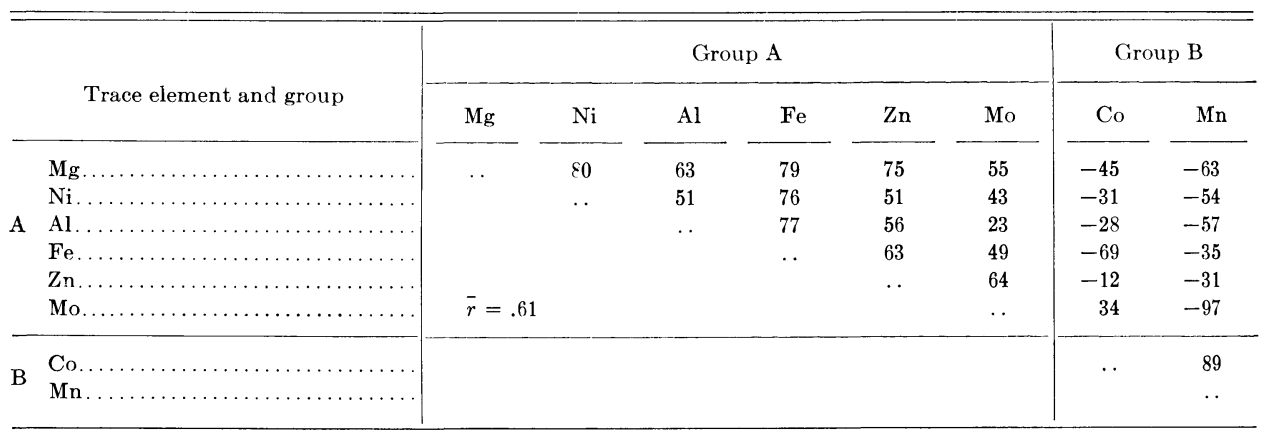

Between A-B, $\bar{r}=-.41$. 
TABLE 5-Continued

Holland Series

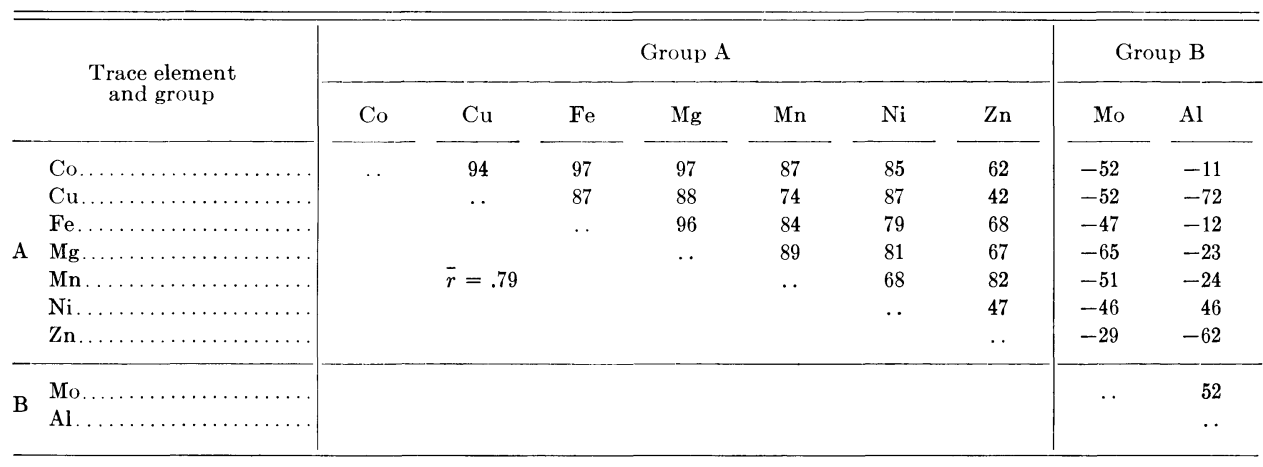

Between $\mathrm{A}-\mathrm{B}, \bar{r}=-.42$.

Los Osos Series

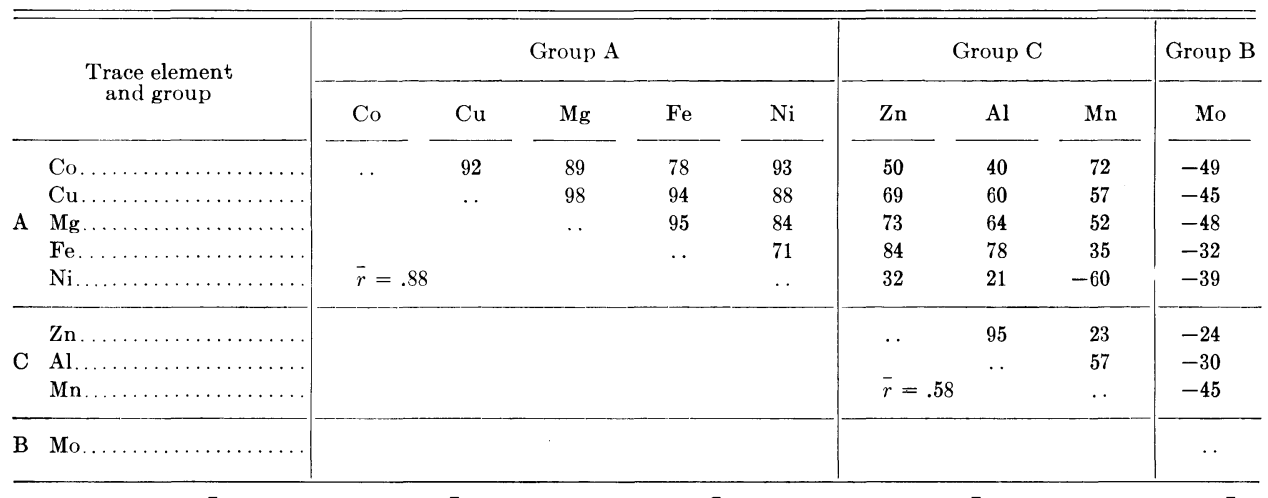

Between A-C, $\bar{r}=.56$; between $\mathrm{A}-\mathrm{B}, \bar{r}=-.43$; between $\mathrm{C}-\mathrm{B}, \bar{r}=.-33 ; \mathrm{AC}$ combined $\bar{r}=.72 ;$ between AC-B, $\bar{r}=$ -39 .

Hanford Series

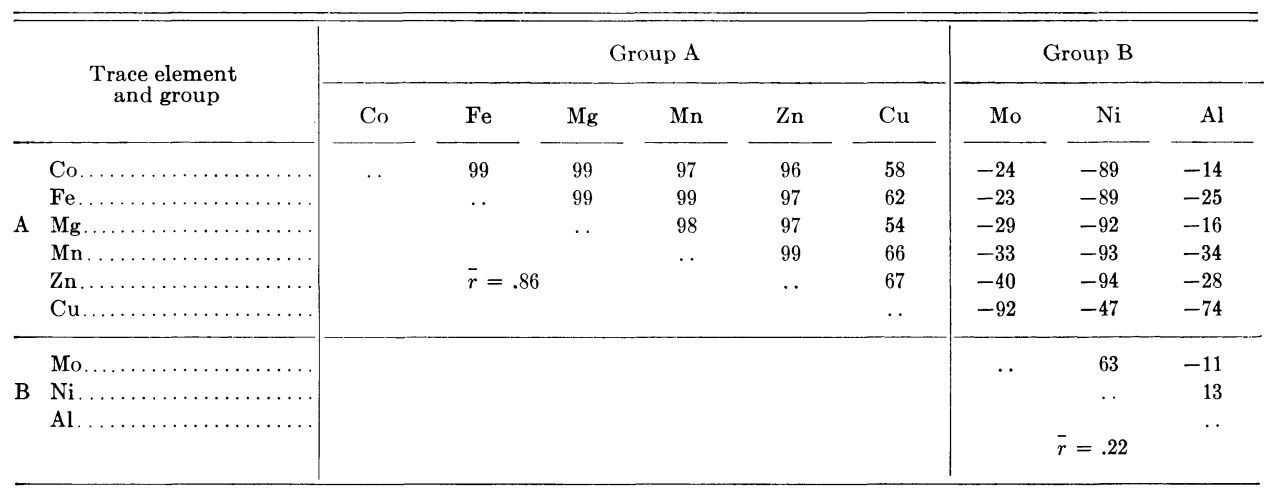

Between $\mathrm{A}-\mathrm{B}, \bar{r}=-.50$. 
TABLE 5-Continued

Ramona Series

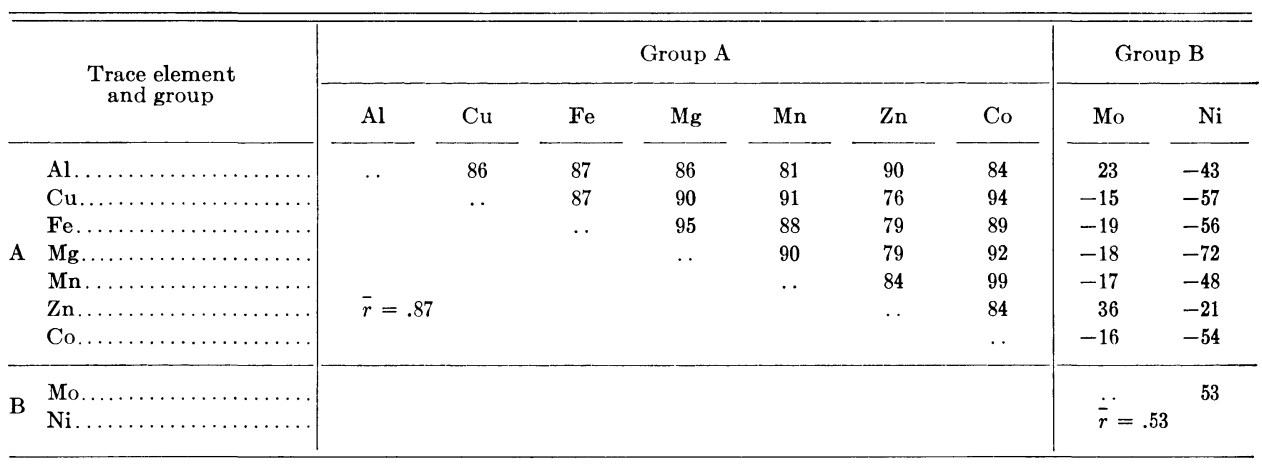

Between A-B, $\bar{r}=-.27$.

Altamont Series

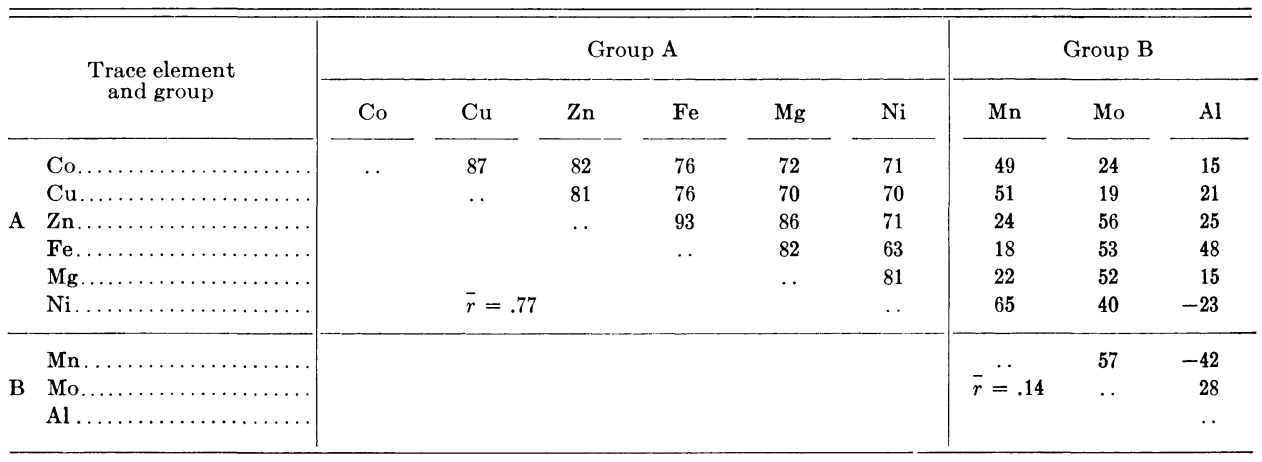

Between A-B, $\bar{r}=32.8$.

San Joaquin Series

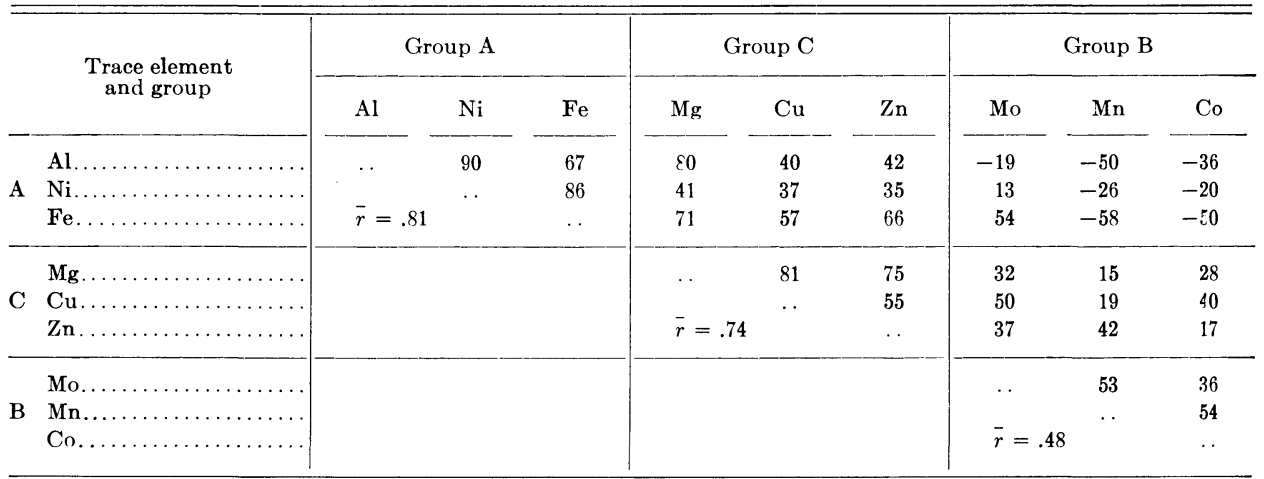

Between $\mathrm{B}-\mathrm{C}, \bar{r}=.31$; between $\mathrm{A}-\mathrm{B}, \bar{r}=-.21$; between $\mathrm{A}-\mathrm{C}, \bar{r}=.52$. 


\section{TABLE 5-Continued}

Fresno Series

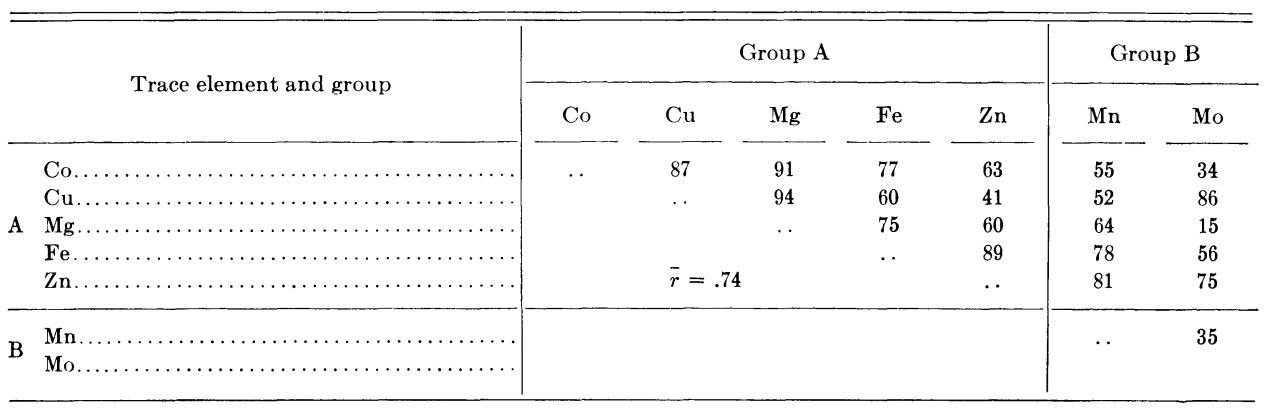

$\mathrm{AB}$ (combined), $\bar{r}=.65$.

Redding Series

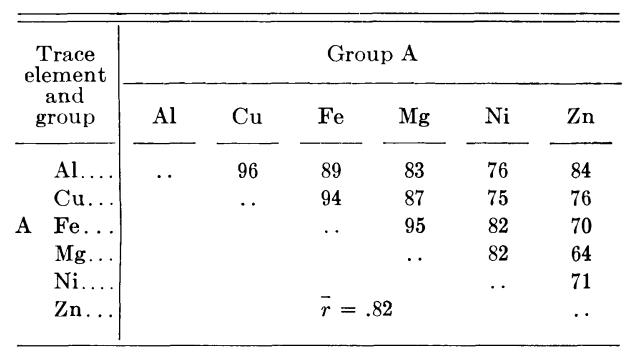

Aiken Series

\begin{tabular}{|c|c|c|c|c|c|}
\hline \multirow{2}{*}{\multicolumn{2}{|c|}{$\begin{array}{l}\text { Trace element } \\
\text { and group }\end{array}$}} & \multicolumn{2}{|c|}{ Group A } & \multicolumn{2}{|c|}{ Group B } \\
\hline & & $\mathrm{Al}$ & Mo & $\mathrm{Mg}$ & Co \\
\hline A & $\begin{array}{l}\text { Al } \ldots \ldots \ldots \\
\text { Mo.......... }\end{array}$ & . & $\begin{array}{l}51 \\
\ldots\end{array}$ & $\begin{array}{l}-18 \\
-68\end{array}$ & $\begin{array}{l}-91 \\
-58\end{array}$ \\
\hline B & $\begin{array}{l}\mathrm{Mg} \ldots \ldots \ldots \\
\mathrm{Co} \ldots \ldots \ldots\end{array}$ & & & $\ldots$ & $\begin{array}{l}93 \\
\cdots\end{array}$ \\
\hline
\end{tabular}

Between $\mathrm{A}-\mathrm{B}, \bar{r}=-.59$.

\section{Hugo Series}

\begin{tabular}{|c|c|c|c|c|c|c|c|}
\hline & \multirow{2}{*}{$\begin{array}{l}\text { Trace element } \\
\text { and group }\end{array}$} & \multicolumn{4}{|c|}{ Group A } & \multicolumn{2}{|c|}{ Group B } \\
\hline & & $\mathrm{Cu}$ & $\mathrm{Fe}$ & $\mathrm{Al}$ & Mo & Mn & $\mathrm{Zn}$ \\
\hline \multirow{4}{*}{ A } & $\mathrm{Cu}$. & & 93 & 57 & 30 & -73 & -18 \\
\hline & $\mathrm{Fe} \ldots \ldots \ldots$ & & .. & 54 & 41 & -72 & -22 \\
\hline & Al... & & & .. & 54 & -63 & -41 \\
\hline & Mo. & $\vec{r}=$. & & & .. & -46 & -15 \\
\hline \multirow{2}{*}{ B } & Mn... & & & & & $\ldots$ & 70 \\
\hline & $\mathrm{Zn} \ldots$ & & & & & & .. \\
\hline
\end{tabular}

Between A-B, $\bar{r}=-.44$. 
TABle 5-Continued

\begin{tabular}{|c|c|c|c|c|c|c|c|c|}
\hline \multirow{2}{*}{\multicolumn{2}{|c|}{$\begin{array}{l}\text { Trace element } \\
\text { and group }\end{array}$}} & \multicolumn{3}{|c|}{ Group A } & \multicolumn{4}{|c|}{ Group B } \\
\hline & & $\mathrm{Cu}$ & $\mathrm{Zn}$ & Mo & $\mathrm{Co}$ & $\mathrm{Mn}$ & $\mathrm{Mg}$ & $\mathrm{Al}$ \\
\hline \multirow{3}{*}{ A } & $\mathrm{Cu} \ldots .$. & & 96 & 92 & -48 & -57 & -69 & 36 \\
\hline & $\mathrm{Zn}$. & & .. & 99 & -62 & -74 & -71 & -47 \\
\hline & Mo. . & $\bar{r}=$. & & .. & -65 & -81 & -67 & -48 \\
\hline \multirow{4}{*}{ B } & Co $\ldots . . . .$. & & & & . & 85 & 51 & 61 \\
\hline & $\mathrm{Mn} . .$. & & & & & .. & 48 & 67 \\
\hline & $\mathrm{Mg} . .$. & & & & \multirow{2}{*}{\multicolumn{2}{|c|}{$\bar{r}=.61$}} & .. & 54 \\
\hline & Al. . & & & & & & & .. \\
\hline
\end{tabular}

Between A-B, $\bar{r}=-.60$.

Maymen Series

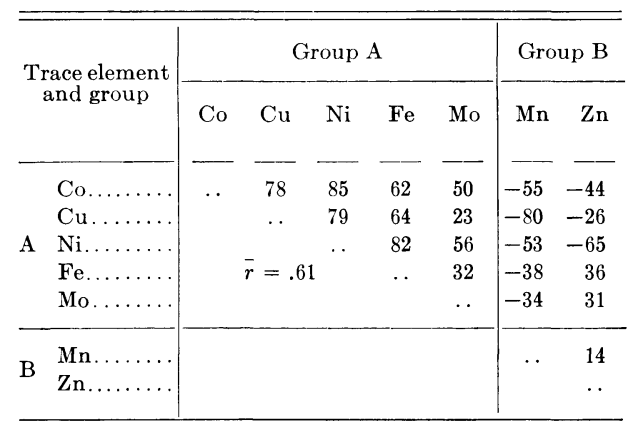

Between A-B, $\bar{r}=.46$.

Mojave Series

\begin{tabular}{|c|c|c|c|c|c|c|c|}
\hline Trace element & $\mathrm{Co}$ & $\mathrm{Cu}$ & $\mathrm{Fe}$ & $\mathrm{Mg}$ & $\mathrm{Ni}$ & $\mathrm{Zn}$ & $\mathrm{Al}$ \\
\hline Co... & . & 79 & 87 & 74 & 90 & 68 & 73 \\
\hline $\mathrm{Cu} \ldots \ldots \ldots \ldots$ & & .. & 89 & 92 & 84 & 70 & 71 \\
\hline $\mathrm{Fe} \ldots \ldots \ldots \ldots$ & & & .. & 94 & 97 & 91 & 71 \\
\hline $\mathrm{Mg}$. & & & & .. & 87 & 90 & 57 \\
\hline $\mathrm{Ni} \ldots \ldots \ldots \ldots$ & & & & & .. & 83 & 78 \\
\hline $\mathrm{Zn} \ldots \ldots \ldots \ldots$ & & & & & & .. & 39 \\
\hline $\mathrm{Al} \ldots \ldots \ldots \ldots$ & & & & & & & . \\
\hline
\end{tabular}

$\bar{r}($ without $\mathrm{Al})=.85 ; \bar{r}($ with $\mathrm{Al})=.79$. 


\section{LITERATURE CITED}

ARCHER, F. C.

1963. Trace elements in some Welsh upland soils. Jour. Soil Sci. 14: 144-48.

BARSHAD, I.

1951. Factors affecting the molybdenum content of pasture plants: I. Nature of soil molybdenum, growth of plants, and soil pH. Soil Sci. 71: 297-313.

Bould, C., D. J. D. Nicholas, J. A. H. Tolmurst, and J. M. S. Potter

1953. Copper deficiency of fruit trees in Great Britian. Jour. Hort. Sci. 28: 268-77.

BRADFORD, G. R., and R. B. HARDING

1964. Severe copper deficiency in orchard grapfruit trees. Hilgardia 35 (12) : 323-27.

Bradford, G. R., P. F. Pratt, F. L. Bair, and B. Goulben

1965. Separation and determination of total $\mathrm{Al}, \mathrm{Mg}, \mathrm{Ni}, \mathrm{Mn}, \mathrm{Co}, \mathrm{Cu}, \mathrm{Fe} \mathrm{Mo}$, and $\mathrm{Zn}$ in soils. Soil Sci. 100 (5) : 309-18.

HARVEY, R. J.

1937. The Denmark wasting disease. Cobalt status of some Western Australia soils. Jour. Dept. Agr. W. Australia 14: 386-92.

KNoтt, J. E.

1933. The effect of certain mineral elements on the color and thickness of onion scales. New York (Cornell) Agr. Exp. Sta. Bul. 552.

Кивота, J.

1964. Cobalt content of New England soils in relation to cobalt levels in forages for ruminant animals. Soil Sei. Soc. Amer. Proc. 28 (2) : 246-51. March-April.

MitChelL, R. L.

1955. Trace elements. In "Chemistry of the Soil," F. E. Bear, Ed. Amer. Chem. Soc. Monograph Ser. No. 126: 253-85.

1963. Soil aspects of trace element problems in plants and animals. Jour. Roy. Agr. Soc. 124: $75-86$.

PatTerson, J. B. E.

1937. Cobalt and sheep diseases. Nature 140: 363.

Purvis, D., and J. M. RAGG.

1962. Copper-deficient soils in Southeast Scotland. Jour. Soil Sei. 13: 241-46.

RIGG, T.

1940. Mineral content of pastures. Cobalt investigations at the Cawthorn Institute. New Zealand Dept. Sci. Ind. Research Ann. Rept. 14: 41-44.

Robinson, W. O., G. Edgington, W. H. Armiger, and A. V. Breen

1951. Availability of molybdenum as influenced by liming. Soil Sci. 72: 267-74.

Russell, Sir. E. J.

1938. Minor elements in plant nutrition. Jour. Roy. Agr. Soc. 99: 331-33.

Stewhrt, J., R. L. Mitchell, and A. B. Stewart

1941. Pining in sheep: its control by administration of cobalt and use of cobalt-rich fertilizers. Empire Jour. Exptl. Agr. 9: 145-52.

STORIE, R. E., and W. W. WEIR

1953. Soil series of California, formation and characteristics, key for identification, pedalogical classifications. Assoc. Students Store, University of California, Berkeley. [Photolith production by the National Press, Palo Alto, Calif.]

SWAINE, D. J.

1955. The trace-element content of soils. Commonwealth Bur. Soil Sci. Tech. Commun. No. 48, Commonwealth Agr. Bur., Herald Printing Works, York, England.

Taylor, N. H., I. J. Cunningham, and E. B. Davies

1956. Soil type in relation to mineral deficiences. Paper No. 32, presented before the 7 th Intern. Grassland Congr., Palmerston North, New Zealand.

Thorne, D. W., W. D. Laws, and A. Wallace

1942. Zine relationships of some Utah soils. Soil Sci. 54: 463-68.

VINK, A. P. A.

1963. Soil survey as related to agricultural productivity. Jour. Soil Sci. 14: 88-101. 
Walsh, T., G. A. Fleming, T. J. Kavanagh, and P. Ryan

1956. Cobalt status of Irish soils and pastures in relation to pining in sheep and eattle. Eire Dept. Agr. Jour. 52: 56-116.

Walsh, T., M. Neenan, and L. B. O'Moore

1952. Molybdenum in relation to cropping and livestock problems under Irish conditions. Nature 170: 149-50.

\section{REFERENCES}

Bedrosian, A. J., and W. J. Hanna

1966. Trace element relationships in New Jersey soils. Soil Sci. 101: 50-56.

OeRTEL, A. C.

1961. Relation between trace element concentrations in soil and parent material. Jour. Soil Sci. 12: 119-28.

Oertel, A. C., and J. B. Giles

1963. Trace element contents of some Queensland soils. Australian Jour. Soil Research 1: $215-22$.

1964. A study of some Brigalow soils based on trace-element profiles. Australian Jour. Soil Research 2: 162-72.

Presant, E. W., and W. M. Tupper

1965. Trace elements in some New Brunswick soils. Canad. Jour. Soil Sci. 45: 305-10.

Sagunov, V. G., and K. O. Nurmagambetov

1965. Supply of trace elements available to plants in soils of some regions of Kazakhstan. Vestn. Akad. Nauk kazakh SSR No. 5: 24-28. 

The journal HILGARDIA is published at irregular intervals, in volumes of about 650 to 700 pages. The number of issues per volume varies.

Single copies of any issue may be obtained free, as long as the supply lasts; please request by volume and issue number from:

$$
\begin{aligned}
& \text { Agricultural Publications } \\
& \text { University Hall } \\
& \text { University of California } \\
& \text { Berkeley, California } 94720
\end{aligned}
$$

The limit to nonresidents of California is 10 separate titles. The limit to California residents is 20 separate titles.

The journal will be sent regularly to libraries, schools, or institutions in one of the following ways:

1. In exchange for similar published material on research.

2. As a gift to qualified repository libraries only.

3. On a subscription basis_ $\$ 7.50$ a year paid in advance. All subscriptions will be started with the first number issued during a calendar year. Subscribers starting dur. ing any given year will be sent back numbers to the first of that year and will be billed for the ensuing year the following January. Make checks or money orders payable to The Regents of The University of California; send payment with order to Agricultural Publications at above address. 